\title{
Characteristic Polynomials of Complex Random Matrix Models
}

\author{
G. Akemann and G. Vernizzi \\ Service de Physique Théorique, CEA/DSM/SPhT Saclay \\ Unité de recherche associée au CNRS \\ F-91191 Gif-sur-Yvette Cédex, France
}

\begin{abstract}
We calculate the expectation value of an arbitrary product of characteristic polynomials of complex random matrices and their hermitian conjugates. Using the technique of orthogonal polynomials in the complex plane our result can be written in terms of a determinant containing these polynomials and their kernel. It generalizes the known expression for hermitian matrices and it also provides a generalization of the Christoffel formula to the complex plane. The derivation we present holds for complex matrix models with a general weight function at finite- $N$, where $N$ is the size of the matrix. We give some explicit examples at finite- $N$ for specific weight functions. The characteristic polynomials in the large- $N$ limit at weak and strong non-hermiticity follow easily and they are universal in the weak limit. We also comment on the issue of the BMN large- $N$ limit.
\end{abstract}

SPhT T02/175

hep-th/0212051 


\section{Introduction}

The study of the spectral statistical properties of random matrix models naturally leads to consider characteristic polynomials of random matrices. Because of the wide applicability of random matrices, the analysis of characteristic polynomials has been approached from different fields of physics and mathematics, such as quantum chaos [1], Quantum Chromodynamics (QCD) [2] or the study of the statistical distribution of the zeros of the Riemann zeta-function 3] to name a few of them.

The expectation value of ratios of random matrix determinants including sources has been introduced in the supersymmetric approach to random matrix models, as it can be used as a generating functional for resolvents and thus for all eigenvalue correlation functions. In the supersymmetric approach the determinants are expressed as integrals over fermionic and bosonic variables which can then be evaluated at finite- $N$ or large- $N$, where $N$ is the size of the matrix. For a review of this approach we refer to [4]. Recently the Ingham-Siegel integral has been used as an alternative to the HubbardStratonovich transformation [5]. Another approach to calculate ratios of characteristic polynomials is the replica method, where new exact results have been obtained very recently by [6] and [7] (see also [8]). A third approach which we will adopt in the following, is the method of orthogonal polynomials. Arbitrary ratios of characteristic polynomials have been calculated by means of this method in [8, 9] and a rigorous proof of their universality has been provided in [10].

In this article we will focus on products of characteristic polynomials. In principle they contain all informations about the eigenvalues. When the matrices are hermitian, products of characteristic polynomials exhibit an interesting duality property, which interchanges the size of the matrix with the number of determinants [1], 12]. Moreover, in the application to QCD they enjoy a direct interpretation as partition functions including quark masses [2]. They also give the orthogonal polynomials and the kernel with respect to a weight function which includes such quark mass terms. Consequently all massive correlation functions can be obtained from them [13]. In fact many of the results for characteristic polynomials have been first obtained in the QCD-related literature in the large- $N$ limit, without explicitly calling them "products of characteristic polynomials" but "massive partition functions". For the unitary ensemble of random matrices they were first calculated in [14] including a universality proof. For the chiral unitary ensemble the partition functions were derived in [15] for finite- $N$ and infinite- $N$ and shown to be universal [16]. For the other symmetry classes of chiral orthogonal and symplectic ensembles the massive partition functions were derived in [17] and [18], and their universality has been proved [18]. The corresponding nonchiral results were obtained in [19]. Results for finite- $N$ and infinite- $N$ were independently found for all three nonchiral symmetry classes in 20] and [11].

Up to now such results were little explored for random matrix models with complex matrices having complex eigenvalues. Our aim is to provide an answer at finite- $N$ and large- $N$ for products of characteristic polynomials where one has to distinguish between the matrix and its hermitian conjugate now. Several developments in various fields have renewed the interest in complex matrix models. Such models have been related for example to the Saffman-Taylor instability on the interface of two-dimensional fluids [21], and correlation functions of complex eigenvalues at large distance were calculated in 22]. The correspondence between supersymmetric Yang-Mills theory and string theory has also renewed the interest in expectation values of traces of complex matrices 23]. There, the complex matrix model belongs to the Ginibre ensemble and is a tool for computing operators in a peculiar large- $N$ limit, the so-called Berenstein-Maldacena-Nastase (BMN) limit [24]. The computational techniques range from combinatorics, character expansion, orthogonal polynomials (as in the original approach of Ginibre [25]), up to large- $N$ loop equation techniques [26] or collective field theory [27].

\footnotetext{
${ }^{1}$ Here we are not considering the relation to finite volume partition functions of QCD, but only to random matrix models partition functions.
} 
Matrices with complex eigenvalues occur also in QCD when a chemical potential for the quarks is introduced 28]. As QCD belongs to the chiral symmetry class, a chiral complex eigenvalue model has been introduced and solved in [29]. The nonchiral complex model with unitary symmetry, corresponding to three-dimensional QCD, was previously studied in [30]. There, the massive partition functions as well as all correlation functions were determined under the hypothesis that the mass terms provide a non-negative definite measure. Here, we will generalize these results to arbitrary products of characteristic polynomials, without any restriction on the sources.

The technique we use in the present article is based on general orthogonal polynomials in the complex plane. Many important properties that usually hold for such polynomials on the real axis (e.g. the existence of a three-step recursion relation or the Christoffel-Darboux formula) are no longer valid in the complex plane in general. Our results will rely solely on the existence of the orthogonal polynomials.

The article is organized as follows. After some definitions we first state and prove our main result in Section 2 for any product of characteristic polynomials of complex matrices and their hermitian conjugate. As a nice consequence we also find a simple determinant expression for products of characteristic polynomials of hermitian matrices, when the number of factors is odd. In Section 3 we give an interpretation of our findings. In some special case they give massive orthogonal polynomials and their kernel, which leads to all massive eigenvalue correlation functions. We also make contact to previous results in [30]. In Section 4 we provide some explicit examples of orthogonal polynomials in the complex plane at finite- $N$. We will also extend the duality [11, 12] between products of characteristic polynomials of different matrix size to complex matrices. Section 5 is devoted to the microscopic large- $N$ limit. The known results for the asymptotics of orthogonal polynomials and their kernel at weak and strong non-hermiticity directly apply to the asymptotics for the characteristic polynomials. Also, the proof of universality at weak non-hermiticity [31] can be extended to our case. We note however that at strong non-hermiticity only the kernel has a smooth microscopic large- $N$ limit (and not the polynomials). Before the conclusions we also briefly comment in Section 6 on the informations about the BMN large- $N$ limit that can be extracted from the products of characteristic polynomials.

\section{Products of characteristic polynomials}

We define our complex matrix model partition function and the corresponding expectation values by

$$
\begin{aligned}
\tilde{\mathcal{Z}}_{N} & \equiv \int\left[d J d J^{\dagger}\right] w\left(J, J^{\dagger}\right), \\
\langle\mathcal{O}\rangle_{N} & \equiv \frac{1}{\tilde{\mathcal{Z}}_{N}} \int\left[d J d J^{\dagger}\right] w\left(J, J^{\dagger}\right) \mathcal{O}\left(J, J^{\dagger}\right),
\end{aligned}
$$

respectively. Here $J$ is a general complex matrix of size $N \times N$ and we integrate over all independent matrix elements. We restrict ourselves to real non-negative weight functions $w\left(J, J^{\dagger}\right)$. In general a complex matrix can be diagonalized by using two unitary matrices. Alternatively it is possible to transform it into a triangular form by a single unitary transformation, the Schur decomposition

$$
J=U(Z+R) U^{-1}, \quad Z=\operatorname{diag}\left(z_{1}, \ldots, z_{N}\right), \quad U \in \mathrm{U}(N), z_{i} \in \mathbb{C},
$$

where $R$ is a strictly upper triangular matrix with complex entries. We assume that the above partition function can be written solely in terms of the complex eigenvalues $z_{i}, i=1, \ldots, N$ and that the weight $w\left(J, J^{\dagger}\right)$ factorizes. We thus can write

$$
\tilde{\mathcal{Z}}_{N}=C(N) \int_{D} \prod_{i=1}^{N}\left(d^{2} z_{i} w\left(z_{i}, \bar{z}_{i}\right)\right)\left|\Delta_{N}(z)\right|^{2} \equiv C(N) \mathcal{Z}_{N}
$$


where we have used that the Jacobian of the transformation (2.3) yields the Vandermonde determinant denoted by

$$
\Delta_{N}(z) \equiv \prod_{i>j}^{N}\left(z_{i}-z_{j}\right)=\operatorname{det}_{1 \leq i, j \leq N}\left[z_{i}^{j-1}\right] .
$$

The constant $C(N)$ contains the integral over the unitary group $U(N)$ as well as the integral over the upper triangular matrix $R$. The latter drops out also in other expectation values, as for example in the determinants we consider below. In eq. (2.4) $D$ denotes the domain in the complex plane over which we integrate the complex eigenvalues. Examples for weight functions satisfying the above conditions $\operatorname{are}^{2}$

$$
w\left(J, J^{\dagger}\right)=\exp \left[\operatorname{tr} V\left(J, J^{\dagger}\right)\right], \quad \text { with } V\left(J, J^{\dagger}\right)=g J J^{\dagger}+V(J)+[V(J)]^{\dagger} .
$$

Here $V(J)$ can be taken for example as an arbitrary complex polynomial and $g$ is a real coupling constant. The domain $D$ can be the full complex plane or any bounded domain, depending on the weight function. For instance if

$$
w\left(J, J^{\dagger}\right)=\delta\left(\operatorname{tr}\left(J J^{\dagger}\right)-r^{2}\right), \quad \text { or } \quad w\left(J, J^{\dagger}\right)=\theta\left(\operatorname{tr}\left(J J^{\dagger}\right)-r^{2}\right),
$$

then the domain is obviously a circle or a disk of radius $r$ around the origin in the complex plane, respectively.

If the weight function $w(z, \bar{z})$ is such that all moments exist, $\int_{D} d^{2} z w(z, \bar{z})|z|^{2 n}<\infty, \forall n \in \mathbb{N}$, then the orthogonal polynomials $\tilde{P}_{n}(z)$

$$
\int_{D} d^{2} z w(z, \bar{z}) \tilde{P}_{k}(z) \overline{\tilde{P}_{l}(z)}=h_{k} \delta_{k l},
$$

exist as well and are uniquely determined by the monic normalization $\tilde{P}_{k}(z)=z^{k}+O\left(z^{k-1}\right)$ with norm $h_{k}$. Note that the coefficients of $\tilde{P}_{l}(z)$ are complex in general. However if both $w(z, \bar{z})$ and the domain $D$ are symmetric under the exchange $z \leftrightarrow \bar{z}$ then $\tilde{P}_{l}(z)$ can be chosen with real coefficients. The theory of orthogonal polynomials in the complex plane (and their asymptotics) is a well-established subject of mathematics; for further details, we refer the interested reader to the mathematical literature (e.g. [32]).

We can easily express the eigenvalue partition function eq. (2.4) in terms of the norms $h_{k}$ by replacing the Vandermonde determinant eq. (2.5) with a determinant of the monic polynomials $\tilde{P}_{k}(z)$ and using the orthogonality relation (2.8):

$$
\mathcal{Z}_{N}=N ! \prod_{k=0}^{N-1} h_{k}
$$

In order to state our results we also introduce the orthonormal polynomials

$$
P_{k}(z) \equiv h_{k}^{-\frac{1}{2}} \tilde{P}_{k}(z)
$$

With these preliminaries we can turn to the main result of this article.

\footnotetext{
${ }^{2}$ In our conventions we will not put a factor of $N$ into the exponent in order to have exact results for finite- $N$. When taking the large- $N$ limit it can be reestablished by scaling $z_{i} \rightarrow \sqrt{N} z_{i}$ and by suitably rescaling the coupling constants in the potential $V$.
} 
ThEorem: Let $\left\{v_{i} ; i=1, \ldots, K\right\}$ and $\left\{u_{i} ; i=1, \ldots, L\right\}$ be two sets of complex numbers which are pairwise distinct among each set. Without loss of generality we assume $K \geq L$, where the empty set with $L=0$ is permitted as well. Together with the definitions and restrictions on the weight function made above, the following statement holds ;

$$
\left\langle\prod_{i=1}^{K} \operatorname{det}\left[v_{i}-J\right] \prod_{j=1}^{L} \operatorname{det}\left[\bar{u}_{j}-J^{\dagger}\right]\right\rangle_{N}=\frac{\prod_{i=N}^{N+K-1} h_{i}^{\frac{1}{2}} \prod_{j=N}^{N+L-1} h_{j}^{\frac{1}{2}}}{\Delta_{K}(v) \Delta_{L}(\bar{u})} \operatorname{det}_{1 \leq l, m \leq K}\left[\mathcal{B}\left(v_{l}, \bar{u}_{m}\right)\right],
$$

where we have defined:

$$
\mathcal{B}\left(v_{l}, \bar{u}_{m}\right) \equiv\left\{\begin{array}{ccl}
\sum_{i=0}^{N+L-1} P_{i}\left(v_{l}\right) \overline{P_{i}\left(u_{m}\right)} & \text { for } & m=1, \ldots, L \\
P_{N+m-1}\left(v_{l}\right) & \text { for } & m=L+1, \ldots, K
\end{array} .\right.
$$

Before presenting the proof of the Theorem let us make a few remarks. The reason why we restrict ourselves to the case $K \geq L$ is that the other case $K \leq L$ can be obtained simply by complex conjugating the above equations. The restriction to pairwise distinct sets of parameters $v_{i}$ or $u_{i}$ can also be lifted. In the limit $v_{i} \rightarrow v_{j}$ the Vandermonde determinant in the denominator vanishes as well as the determinant in the numerator. Hence in this limit the row containing $v_{j}$ has to be differentiated with respect to $v_{j}$ and then we set $v_{j}=v_{i}$ there. The same procedure can be iterated when $v_{i}$ is degenerate of order $m$. The result is given by substituting the $k$-th row of eq. (2.12) for $k=1, \ldots, m$ with its $(k-1)$-th derivative at $v_{i}$ (and dropping the corresponding arguments in the Vandermonde determinants in the denominator). The same argument applies for the case with degenerate $\bar{u}_{i}$. We will give an example for $L=0$ in eq. (4.22).

We also wish to mention that the object occurring in the first $L$ columns of the matrix $\mathcal{B}\left(v_{l}, \bar{u}_{m}\right)$ is, apart from the weight factors, nothing else than the kernel of orthogonal polynomials defined as:

$$
K_{N}(v, \bar{u}) \equiv[w(v, \bar{v}) w(u, \bar{u})]^{\frac{1}{2}} \sum_{i=0}^{N-1} P_{i}(v) \overline{P_{i}(u)} \equiv[w(v, \bar{v}) w(u, \bar{u})]^{\frac{1}{2}} \kappa_{N}(v, \bar{u}) .
$$

Here we have also explicitly defined the bare kernel $\kappa_{N}(v, \bar{u})$ as it occurs in the Theorem and subsequent formulas. We note that this kernel cannot be written in terms of polynomials of order $N$ and $N-1$ alone because for orthogonal polynomials in the complex plane the analog of the Christoffel-Darboux formula, eq. (2.18) below, does not hold in general (for an example see [31]).

Proof: The proof will be done in two steps. First we will prove the case $K=L$ for arbitrary $L$. Then we consider the case $K>L$ using induction. For $K=L$ we have:

$$
\begin{aligned}
& \left\langle\prod_{i=1}^{L} \operatorname{det}\left[v_{i}-J\right] \prod_{j=1}^{L} \operatorname{det}\left[\bar{u}_{j}-J^{\dagger}\right]\right\rangle_{N}= \\
& \quad=\frac{1}{\mathcal{Z}_{\mathcal{N}}} \int_{D} \prod_{i=1}^{N}\left(d^{2} z_{i} w\left(z_{i}, \bar{z}_{i}\right) \prod_{j=1}^{L}\left(\left(v_{j}-z_{i}\right)\left(\bar{u}_{j}-\bar{z}_{i}\right)\right)\right) \Delta_{N}(z) \Delta_{N}(\bar{z})
\end{aligned}
$$

\footnotetext{
${ }^{3}$ The following notation is understood: $\Delta_{0}(x)=\Delta_{1}(x)=1$ and $\prod_{i=N}^{N-1} h_{i}=1$
} 


$$
\begin{aligned}
& =\frac{1}{\mathcal{Z}_{N}} \int_{D}\left(\prod_{i=1}^{N} d^{2} z_{i} w\left(z_{i}, \bar{z}_{i}\right)\right) \frac{\Delta_{N+L}\left(z_{1}, \ldots, z_{n}, v_{1}, \ldots, v_{L}\right)}{\Delta_{L}\left(v_{1}, \ldots, v_{L}\right)} \frac{\Delta_{N+L}\left(\bar{z}_{1}, \ldots, \bar{z}_{n}, \bar{u}_{1}, \ldots, \bar{u}_{L}\right)}{\Delta_{L}\left(\bar{u}_{1}, \ldots, \bar{u}_{L}\right)} \\
& =\frac{1}{\mathcal{Z}_{N} \Delta_{L}(v) \Delta_{L}(\bar{u})} \int_{D} \prod_{i=1}^{N}\left(d^{2} z_{i} w\left(z_{i}, \bar{z}_{i}\right)\right) \operatorname{det}_{1 \leq i, j \leq N+L}\left[\zeta_{i}^{j-1}\right] \operatorname{det}_{1 \leq i, j \leq N+L}\left[\bar{\eta}_{i}^{j-1}\right], \text { with }\left\{\begin{array}{l}
\zeta \equiv\{z, v\} \\
\eta \equiv\{z, u\}
\end{array}\right. \\
& =\frac{\prod_{i=0}^{N+L-1} h_{i}}{\mathcal{Z}_{N} \Delta_{L}(v) \Delta_{L}(\bar{u})} \int_{D} \prod_{i=1}^{N}\left(d^{2} z_{i} w\left(z_{i}, \bar{z}_{i}\right)\right) \operatorname{det}_{1 \leq i, j \leq N+L}\left[P_{j-1}\left(\zeta_{i}\right)\right] \operatorname{det}_{1 \leq i, j \leq N+L}\left[\overline{P_{j-1}\left(\eta_{i}\right)}\right] \\
& =\frac{\prod_{i=N}^{N+L-1} h_{i}}{N ! \Delta_{L}(v) \Delta_{L}(\bar{u})} \int_{D} \prod_{i=1}^{N}\left(d^{2} z_{i} w\left(z_{i}, \bar{z}_{i}\right)\right) \operatorname{det}_{1 \leq i, j \leq N+L}\left[\sum_{r=1}^{N+L} P_{r-1}\left(\zeta_{i}\right) \overline{P_{r-1}\left(\eta_{j}\right)}\right] .
\end{aligned}
$$

In the second step we have put the products of differences between $v_{j}$ and $z_{i}$ into a larger Vandermonde determinant of size $N+L$ and between $\bar{u}_{j}$ and $\bar{z}_{i}$ into a complex conjugated one. The additional factors of differences between $v_{j}$ 's and $\bar{u}_{j}$ 's have been divided out in terms of smaller Vandermonde determinants of size $L$. The Vandermonde determinant can be written as a determinant of powers, eq. (2.5), where we have introduced a larger set of variables $\zeta_{1, \ldots, N+L}$ and $\eta_{1, \ldots, N+L}$. The properties of determinants allow to replace the powers $\zeta_{i}^{j-1}$ first by the monic polynomials $\tilde{P}_{j-1}\left(\zeta_{i}\right)$ and then by the orthonormal ones times their norms, which have been taken out of the determinants. They partially cancel the normalization $\mathcal{Z}_{N}$ from eq. (2.9). We then apply that $\operatorname{det}[A] \operatorname{det}[B]=\operatorname{det}\left[A B^{T}\right]$ and thus obtain a single determinant of the bare kernel eq. (2.13). Now we make use of Theorem 5.2.1 in [33], which can be applied as follows. The kernel $K_{N}(\zeta, \bar{\eta})$ satisfies the hermiticity condition $\overline{K_{N}(\zeta, \bar{\eta})}=K_{N}(\eta, \bar{\zeta})$ and the chain relation $\int d^{2} z K_{N}(\zeta, \bar{z}) K_{N}(z, \bar{\eta})=K_{N}(\zeta, \bar{\eta})$. Then

$$
\int d^{2} z_{n} \underset{1 \leq i, j \leq n}{\operatorname{det}}\left[K_{N}\left(z_{i}, \bar{z}_{j}\right)\right]=(c-n+1) \operatorname{det}_{1 \leq i, j \leq n-1}\left[K_{N}\left(z_{i}, \bar{z}_{j}\right)\right]
$$

where $c=\int d^{2} z K_{N}(z, \bar{z})=N$. We can thus successively integrate out all $z_{1, \ldots, N}$ using eq. (2.15). The successive use produces a factor $N$ ! and we thus arrive precisely at eq. (2.11) for $K=L$, with the matrix $\mathcal{B}$ only consisting of the bare kernels.

Next we prove eq. (2.11) for $K>L$ by induction in $K-L$. The starting point of induction is already satisfied for $K=L$. For the induction step $K \rightarrow K+1$ we proceed as follows:

$$
\begin{aligned}
& \left\langle\prod_{i=1}^{K+1} \operatorname{det}\left[v_{i}-J\right] \prod_{j=1}^{L} \operatorname{det}\left[\bar{u}_{j}-J^{\dagger}\right]\right\rangle_{N}= \\
& =\frac{1}{\mathcal{Z}_{N} \Delta_{K+1}(v) \Delta_{L}(\bar{u})} \int_{D} \prod_{i=1}^{N} d^{2} z_{i} w\left(z_{i}, \bar{z}_{i}\right) \\
& \times \sum_{\sigma \in\{0, \ldots, N+K\}}(-1)^{\sigma} \tilde{P}_{\sigma(1)}\left(z_{1}\right) \cdots \tilde{P}_{\sigma(N)}\left(z_{N}\right) \tilde{P}_{\sigma(N+1)}\left(v_{1}\right) \cdots \tilde{P}_{\sigma(N+K)}\left(v_{K}\right) \tilde{P}_{\sigma(N+K+1)}\left(v_{K+1}\right) \\
& \times \sum_{\sigma^{\prime} \in\{0, \ldots, N+L-1\}}(-1)^{\sigma^{\prime}} \overline{\tilde{P}_{\sigma^{\prime}(1)}\left(z_{1}\right)} \cdots \overline{\tilde{P}_{\sigma^{\prime}(N)}\left(z_{N}\right)} \overline{\tilde{P}_{\sigma^{\prime}(N+1)}\left(u_{1}\right)} \cdots \overline{\tilde{P}_{\sigma^{\prime}(N+L)}\left(u_{L}\right)} \\
& =\frac{\prod_{i=N}^{N+K} h_{i}^{\frac{1}{2}} \prod_{j=N}^{N+L-1} h_{j}^{\frac{1}{2}}}{\Delta_{K+1}(v) \Delta_{L}(\bar{u})}\left(P_{N+K}\left(v_{K+1}\right) \operatorname{det}_{1 \leq l, m \leq K}\left[\mathcal{B}\left(v_{l}, \bar{u}_{m}\right)\right]\right. \\
& \left.-\sum_{j=1}^{K} P_{N+K}\left(v_{j}\right) \underset{\substack{m=1, \ldots, K \\
l=1, \ldots, j-1, K+1, j+1, \ldots, K}}{\operatorname{det}}\left[\mathcal{B}\left(v_{l}, \bar{u}_{m}\right)\right]\right) \text {. }
\end{aligned}
$$


We repeated the first steps in eq. (2.14) until we have the product of two determinants of monic polynomials and their conjugate, containing the variables $\left\{z_{1, \ldots, N}, v_{1, \ldots, K+1}\right\}$ and $\left\{z_{1, \ldots, N}, \bar{u}_{1, \ldots, L}\right\}$, respectively. We have explicitly spelled out these determinants as sums over permutations. The index $\sigma(N+K+1)$ of the polynomial $\tilde{P}_{\sigma(N+K+1)}\left(v_{K+1}\right)$ can take values in $\{0, \ldots, N+K\}$. For $\sigma(N+K+1)=N+K$ the polynomial $\tilde{P}_{N+K}\left(v_{K+1}\right)$ multiplies exactly the expectation value $\left\langle\prod_{i=1}^{K} \operatorname{det}\left[v_{i}-J\right] \prod_{j=1}^{L} \operatorname{det}\left[\bar{u}_{j}-J^{\dagger}\right]\right\rangle_{N}$ for which the Theorem holds by using the assumption of induction. The other values for the index $\sigma(N+K+1)<N+K$ can be obtained by simply exchanging the argument $v_{K+1}$ with the one of the corresponding polynomial of argument $v_{j=1, \ldots, K}$. Since this is a pair permutation in $\sigma$ it will produce a sign for all terms. As a final step, in the last line of eq. (2.16) we move the row containing $v_{K+1}$ from the position $j$ to the lowest row of position $K$, without changing the order of the other rows in the determinants. This produces a factor $(-1)^{K-j}$. Then we see that the sum of all determinants is just the Laplace expansion of a matrix of size $K+1$ with respect to the last column, and that this matrix is nothing else than $\mathcal{B}\left(v_{l}, \bar{u}_{m}\right)$ in eq. (2.12). We have thus proved the Theorem for $K+1$.

Let us emphasize the Theorem (2.11) also holds for partition functions defined as eigenvalue integrals such as eq. (2.4) and which do not necessarily have a representation in terms of complex matrices. Examples for such models are the chiral complex matrix model as recently proposed in [29] which we will discuss again in Section 4 . For this model no explicit matrix realization is known so far. Another example are weight functions containing mixed higher powers of $z$ and $\bar{z}$ such as $w(z, \bar{z})=\exp \left[-(z \bar{z})^{k \geq 2}\right]$. Such weight functions can be only written down in terms of complex matrices which are normal, $\left[J, J^{\dagger}\right]=0$, as normal matrices can be diagonalized without an upper triangular matrix as in eq. (2.3). However, such terms with higher powers in $|z|^{2}$ may be necessary in the weight function to write down convergent integrals.

After having completed the proof let us compare to the known results for products of characteristic polynomials of hermitian matrices $J=H=H^{\dagger}$. Since in our proof we have only assumed the existence of an eigenvalue representation for the partition function and the existence of orthogonal polynomials on some domain $D$, it holds also for arbitrary hermitian matrix models. We only have to restrict $D$ to the real line and replace complex integrals $\int d^{2} z$ by real integrals $\int d x$. Because of $J=J^{\dagger}$ the splitting into two sets of determinants becomes immaterial here. In particular for $L=0$ we find back the known result (see e.g. in [20, 11, 12])

$$
\left\langle\prod_{i=1}^{K} \operatorname{det}\left[v_{i}-H\right]\right\rangle_{N}=\frac{1}{\Delta_{K}(v)} \operatorname{det}_{1 \leq l, m \leq K}\left[\tilde{P}_{N+m-1}\left(v_{l}\right)\right] .
$$

The product of $K$ characteristic polynomials is thus given by a $K \times K$ determinant of the orthogonal polynomials. In [10] it was shown that for even $K=2 k$ this can be further simplified to a $k \times k$ determinant. We obtain the same result from setting $K=L=k$ in our Theorem (2.11) and using that for orthogonal polynomials on the real line the Christoffel-Darboux identity holds,

$$
\sum_{i=0}^{N-1} P_{i}(x) P_{i}(y)=\sqrt{\frac{h_{N}}{h_{N-1}}} \frac{P_{N}(x) P_{N-1}(y)-P_{N}(y) P_{N-1}(x)}{x-y},
$$


with $x, y$ real numbers. We thus have

$$
\begin{aligned}
& \left\langle\prod_{i=1}^{k}\left(\operatorname{det}\left[v_{i}-H\right] \operatorname{det}\left[u_{i}-H\right]\right)\right\rangle_{N}= \\
& \quad=\frac{\prod_{i=N}^{N+k-1} h_{i}}{\left(h_{N+k-1}\right)^{k} \Delta_{k}(v) \Delta_{k}(u)} \operatorname{det}_{1 \leq l, m \leq k}\left[\frac{\tilde{P}_{N+k}\left(v_{l}\right) \tilde{P}_{N+k-1}\left(u_{m}\right)-\tilde{P}_{N+k}\left(u_{m}\right) \tilde{P}_{N+k-1}\left(v_{l}\right)}{v_{l}-u_{m}}\right] .
\end{aligned}
$$

As a simple corollary we can thus show from the Theorem (2.11) that also for and odd number $K=2 k+1$ of products of characteristic polynomials the $K \times K$ determinant in eq. (2.17) can be reduced down to size $(k+1) \times(k+1)$.

COROLlary: Let the definitions $(2.1)-(2.5)$ and $(2.8)-(2.10)$ be valid as well for hermitian matrices $H$ with real eigenvalues integrated over a real domain $D$. Take the parameters (real or complex) $\left\{v_{i} ; i=1, \ldots, k+1\right\}$ and $\left\{u_{i} ; i=1, \ldots, k\right\}$ to be all pairwise distinct. From Theorem (2.11) the following relation follows:

$$
\left\langle\prod_{i=1}^{k+1} \operatorname{det}\left[v_{i}-H\right] \prod_{j=1}^{k} \operatorname{det}\left[u_{j}-H\right]\right\rangle_{N}=\frac{\prod_{i=N}^{N+k-1} h_{i}}{\left(h_{N+k-1}\right)^{k} \Delta_{k+1}(v) \Delta_{k}(u)} \operatorname{det}_{1 \leq l, m \leq k+1}\left[\mathbf{B}\left(v_{l}, u_{m}\right)\right],
$$

where we have defined

$$
\mathbf{B}\left(v_{l}, u_{m}\right) \equiv\left\{\begin{array}{cc}
\frac{\tilde{P}_{N+k}\left(v_{l}\right) \tilde{P}_{N+k-1}\left(u_{m}\right)-\tilde{P}_{N+k}\left(u_{m}\right) \tilde{P}_{N+k-1}\left(v_{l}\right)}{v_{l}-u_{m}} & \text { for } m=1, \ldots, k \\
\tilde{P}_{N+m-1}\left(v_{l}\right) & \text { for } \quad m=k+1
\end{array} .\right.
$$

It is clear that from Theorem (2.11) for real hermitian matrices we can get many equivalent formulas for determinants of size in between eq. (2.17) and eqs. (2.19) or (2.20) respectively, by splitting the total number of parameters in different pairs $K$ and $L$.

\section{Applications}

In this Section we give an interpretation for some particular products of characteristic polynomials we calculated in eq. (2.11). From the beginning of our investigations we have assumed that the weight function has to be non-negative definite on the domain $D$ in order to make the construction of the polynomials via Gram-Schmidt possible. The examples given for $w(z, \bar{z})$ in eqs. (2.6) and (2.7) obviously satisfy this requirement. We will now use the results from our Theorem to explicitly construct orthogonal polynomials for more complicated weight functions containing determinants or in other word weights with products of eigenvalues as prefactors.

From the theory of orthogonal polynomials defined on the real line the following is known. Choosing any parameter $v_{j} \in \mathbb{R}$ in eq. (2.17) for hermitian matrices the right hand side can be interpreted as a polynomial in $v_{j}$ of order $N, \vec{P}_{N}\left(v_{j}\right)$, which is orthogonal with respect to the weight $w^{(K-1)}(x) \equiv$

\footnotetext{
${ }^{4}$ Formula (2.18) holds algebraically also for $x, y \in \mathbb{C}$ and $P_{k}(x)$ being the analytic continuation to the complex plane of the polynomials which are orthonormal on the real line. However we emphasize that in this case the sum $\sum_{i=0}^{N-1} P_{i}(x) P_{i}(y)$ is not a kernel in the complex plane, because the $P_{k}$ are not longer orthonormal in the complex plane (for an explicit example see eq. (4.6) ).
} 
$w(x) \prod_{i \neq j}\left(v_{i}-x\right)$. This holds provided that the new weight is non-negative definite on the domain $D$. Note that under this condition, eq. (2.17) immediately follows from the Christoffel Theorem [34]. An example is when $D \subseteq \mathbb{R}$ and all $v_{i}$ are purely imaginary, coming in complex conjugate pairs. This would correspond to the weight for the random matrix partition function of QCD in three dimensions.

In order to make such an interpretation of the characteristic polynomial in the complex plane possible we have to choose $K=L+1$ in eq. (2.11) and to take all $u_{i}=v_{i}, i=1, \ldots, L$. It is easy to convince oneself by going to an eigenvalue basis that the following matrix representation holds:

$$
\tilde{P}_{N}^{(L)}(z) \sim\left\langle\operatorname{det}[z-J] \prod_{i=1}^{L}\left(\operatorname{det}\left[v_{i}-J\right] \operatorname{det}\left[\bar{v}_{i}-J^{\dagger}\right]\right)\right\rangle_{N} .
$$

This gives a polynomial orthogonal with respect to the non-negative definite weight

$$
w^{(L)}\left(J, J^{\dagger}\right) \equiv w\left(J, J^{\dagger}\right) \prod_{i=1}^{L}\left(\operatorname{det}\left[v_{i}-J\right] \operatorname{det}\left[\bar{v}_{i}-J^{\dagger}\right]\right),
$$

and the complex conjugate polynomial $\overline{\tilde{P}_{N}^{(L)}(z)}$ is simply obtained by taking the hermitian conjugate of eq. (3.1). Please note the exact analogy with a similar formula for orthogonal polynomials on the real line (see e.g. [34). In order to satisfy the normalization $\tilde{P}_{N}^{(L)}(z)=z^{N}+\ldots$ we define

$$
\tilde{P}_{N}^{(L)}(z) \equiv \frac{\left\langle\operatorname{det}[z-J] \prod_{i=1}^{L}\left(\operatorname{det}\left[v_{i}-J\right] \operatorname{det}\left[\bar{v}_{i}-J^{\dagger}\right]\right)\right\rangle_{N}}{\left\langle\prod_{i=1}^{L}\left(\operatorname{det}\left[v_{i}-J\right] \operatorname{det}\left[\bar{v}_{i}-J^{\dagger}\right]\right)\right\rangle_{N}} .
$$

Let us give an example for $L=1$ :

$$
\tilde{P}_{N}^{(1)}(z)=\frac{\kappa_{N+1}(z, \bar{v}) \tilde{P}_{N+1}(v)-\kappa_{N+1}(v, \bar{v}) \tilde{P}_{N+1}(z)}{(v-z) \kappa_{N+1}(v, \bar{v})},
$$

where we have used the bare kernel $\kappa_{N+1}(v, \bar{u})$ eq. (2.13) and the superscript $(L=0)$ has been dropped. We can also calculate the norm of the polynomial given by

$$
h_{N}^{(1)}=h_{N+1} \frac{\kappa_{N+2}(v, \bar{v})}{\kappa_{N+1}(v, \bar{v})},
$$

and we obtain for the orthonormal polynomials

$$
P_{N}^{(1)}(z)=\frac{\kappa_{N+1}(z, \bar{v}) P_{N+1}(v)-\kappa_{N+1}(v, \bar{v}) P_{N+1}(z)}{(v-z) \sqrt{\kappa_{N+1}(v, \bar{v}) \kappa_{N+2}(v, \bar{v})}} .
$$

We have not been able to prove a similar expression for the orthonormal polynomials at general $L$. We expect to obtain the following result for the norm of $\tilde{P}_{N}^{(L)}(z)$ :

$$
h_{N}^{(L)}=h_{N+L} \frac{\operatorname{det}_{i, j=1, \ldots, L}\left[\kappa_{N+L+1}\left(v_{i}, \bar{v}_{j}\right)\right]}{\operatorname{det}_{i, j=1, \ldots, L}\left[\kappa_{N+L}\left(v_{i}, \bar{v}_{j}\right)\right.},
$$

which we have checked for $L=1,2,3,4,5$. This would give us $P_{N}^{(L)}(z)$ in a form very similar to [35].

After having determined the orthogonal polynomials for weight functions $w^{(L)}\left(J, J^{\dagger}\right)$ we can use them to obtain the respective kernel as defined in eq (2.13):

$$
K_{N}^{(L)}(z, \bar{u})=\left[w^{(L)}(z, \bar{z}) w^{(L)}(u, \bar{u})\right]^{\frac{1}{2}} \sum_{i=0}^{N-1} P_{i}^{(L)}(z) \overline{P_{i}^{(L)}(u)} .
$$


While we have an explicit expression for $L=1$ in eq. (3.6) we would still have to calculate the norms $h_{N}^{(L)}$ of the polynomials $\tilde{P}_{N}^{(L)}(z)$ which are of the conjectured form eq. (3.7). However, we can also directly read off the kernel from the Theorem (2.11). It is known that not only the polynomials have a matrix representation as in eq. (3.3) but also the kernel itself. Choosing the same weight eq. (3.2) one has to insert two more determinants instead of only one as for the polynomials:

$$
K_{N}^{(L)}(z, \bar{u})=\left[w^{(L)}(z, \bar{z}) w^{(L)}(u, \bar{u})\right]^{\frac{1}{2}} \frac{\left\langle\operatorname{det}[z-J] \operatorname{det}\left[\bar{u}-J^{\dagger}\right] \prod_{i=1}^{L}\left(\operatorname{det}\left[v_{i}-J\right] \operatorname{det}\left[\bar{v}_{i}-J^{\dagger}\right]\right)\right\rangle_{N-1}}{h_{N}\left\langle\prod_{i=1}^{L}\left(\operatorname{det}\left[v_{i}-J\right] \operatorname{det}\left[\bar{v}_{i}-J^{\dagger}\right]\right)\right\rangle_{N}} .
$$

This can be shown along the same lines as in [36] where the same statement was made for hermitian matrices. Note the different matrix size of the expectation value for numerator and denominator. We also give the example with $L=1$ explicitly as it follows from eq. (2.11):

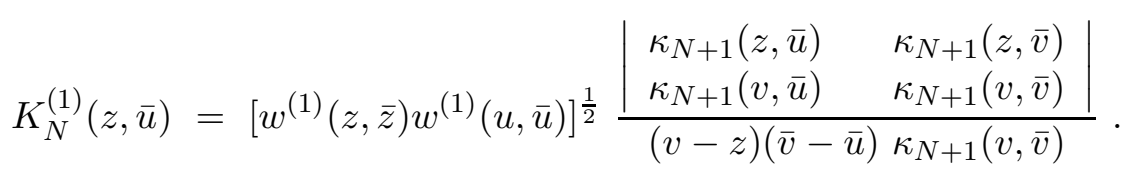

While eqs. (3.8) and (3.9) are equivalent by definition, for general $L$ it is easy to see that already for $L=1$ this yields a highly nontrivial identity, comparing eq. (3.10) and eq. (3.8) with the polynomials eq. (3.6) inserted. From a practical point of view the second form eq. (3.9) which can be directly deduced from the Theorem (2.11) is much more convenient as it is only a ratio of two determinants. Compared to this in eq. (3.8) we have to perform a sum over ratios of determinants of growing size.

Usually the aim of calculating orthogonal polynomials and their kernel is to evaluate correlation functions of eigenvalues defined as

$$
R_{N}^{(L)}\left(z_{1}, \ldots, z_{k}\right) \equiv \frac{1}{\mathcal{Z}_{N}} \frac{N !}{(N-k) !} \int_{D} \prod_{l=k+1}^{N} d^{2} z_{l} \prod_{i=1}^{N} w^{(L)}\left(z_{i}, \bar{z}_{i}\right)\left|\Delta_{N}(z)\right|^{2} .
$$

Using standard orthogonal polynomials techniques [33] they are given by

$$
R_{N}^{(L)}\left(z_{1}, \ldots, z_{k}\right)=\operatorname{det}_{i, j=1, \ldots, k}\left[K_{N}^{(L)}\left(z_{i}, \bar{z}_{j}\right)\right] .
$$

Therefore from eq. (3.9) we can immediately read off all $k$-point correlation functions. They are given by a $k \times k$ determinant of the kernel $K_{N}^{(L)}\left(z_{i}, \bar{z}_{j}\right)$ which is itself again a ratio of determinants of size $(L+1) \times(L+1)$ over $L \times L$. There is an even more compact expression for the $k$-point functions which has been introduced in [30]. It uses the fact that the correlation functions with weight $w^{(L)}\left(J, J^{\dagger}\right)$ from eq. (3.2) can be entirely expressed in terms of higher $n$-point correlation functions with weight $w\left(J, J^{\dagger}\right)$ at $L=0$ 尼:

$$
R_{N}^{(L)}\left(z_{1}, \ldots, z_{k}\right)=\frac{R_{N+L}^{(0)}\left(z_{1}, \ldots, z_{k}, v_{1}, \ldots, v_{L}\right)}{R_{N+L}^{(0)}\left(v_{1}, \ldots, v_{L}\right)}
$$

This result reduces the $k$-point correlation function to a single ratio of determinants of size $(k+L) \times$ $(k+L)$ over $L \times L$. While for the 1-point function $R_{N}^{(L)}(z)=K_{N}^{(L)}(z, \bar{z})$ it is easy to see that eq. (3.13) and eq. (3.12) together with eq. (3.9) agree perfectly, for higher $(k>2)$-point functions their equivalence requires highly nontrivial identities among determinants. The fact that several different (but equivalent) determinant formulations exist, depending on the way they are derived, is a common phenomenon also for correlations functions of hermitian matrices. In some cases their equivalence can be directly shown 37] (see also 38]).

\footnotetext{
${ }^{5}$ Note the typo in the index of the denominator in eq. (2.12) of ref. 30].
} 


\section{Explicit results for finite- $N$}

In the first subsection we will give some explicit examples for weight functions and their corresponding orthogonal polynomials and kernel at finite- $N$. These are the objects that then have to be inserted into Theorem eq. (2.11). In particular our second example will be studied also in Section 5, when taking the large- $N$ limit. In the second subsection we derive a finite- $N$ duality relation between characteristic polynomials of different matrix size.

\subsection{Examples for orthogonal polynomials in the complex plane}

We start with the Ginibre ensemble where the weight function is defined on the full complex plane $D=\mathbb{C}$ as

$$
w_{G}\left(J, J^{\dagger}\right) \equiv \frac{1}{\pi} \exp \left[-\operatorname{tr} J J^{\dagger}\right]=\frac{1}{\pi} \prod_{i=1}^{N} \exp \left[-z_{i} \bar{z}_{i}\right] .
$$

As one can easily convince oneself the orthogonal polynomials are monic powers,

$$
\tilde{P}_{j}(z)=z^{j}, \quad h_{j}=j !
$$

We thus have from the orthonormal polynomials $P_{j}(z)=z^{j}(j !)^{-\frac{1}{2}}$ a simple expression for the kernel

$$
\kappa_{N}(v, \bar{u})=\sum_{i=0}^{N-1} \frac{(v \bar{u})^{i}}{i !}=\exp [v \bar{u}] \frac{\Gamma(N, v \bar{u})}{\Gamma(N)},
$$

where $\Gamma(a, z)=\int_{z}^{\infty} d t t^{a-1} e^{-t}$ is the incomplete gamma function. As a consequence of the results in Section 3 we can also immediately read off explicitly the polynomials for the weight function $w_{G}^{(L)}(z, \bar{z})=\prod_{i=1}^{L} \operatorname{det}\left[\left(v_{i}-z\right)\left(\bar{v}_{i}-\bar{z}\right)\right] \exp [-z \bar{z}]$ from eq. (3.3). For $L=1$ they are given by:

$$
\begin{aligned}
\tilde{P}_{N}^{(1)}(z) & =\frac{v^{N+1} \sum_{i=0}^{N} \frac{(z \bar{v})^{i}}{h_{i}}-z^{N+1} \sum_{i=0}^{N} \frac{(v \bar{v})^{i}}{h_{i}}}{(v-z) \sum_{i=0}^{N} \frac{(v \bar{v})^{i}}{h_{i}}} \\
& =v^{N} \sum_{j=0}^{N}\left(\frac{z}{v}\right)^{j} \frac{\kappa_{j+1}(v, \bar{v})}{\kappa_{N+1}(v, \bar{v})} .
\end{aligned}
$$

Note that eq. (4.4) holds for any $h_{i}$, and not just for $h_{i}=i$ !. Moreover note that we started from polynomials for $L=0$ with real coefficients eq. (4.2) while those of the new polynomials with $L=1$ are now complex. This example shows a remarkable fact. It is well-known that when $D$ is a real interval there is a correspondence between the distribution of the eigenvalues and the distribution of the zeros of the orthogonal polynomials. However in the complex plane such a correspondence breaks down. For instance, eq. (4.2) shows that all the zeros are at the origin $z=0$, whereas the spectral density spreads into the full complex plane.

The following example is for the weight

$$
w_{H}\left(J, J^{\dagger}\right) \equiv \exp \left[-\frac{1}{1-\tau^{2}} \operatorname{tr}\left(J J^{\dagger}-\frac{\tau}{2}\left(J^{2}+J^{\dagger 2}\right)\right)\right], \quad \tau \in[0,1],
$$

which is again defined on $D=\mathbb{C}$. Here the complex matrix is parameterized as $J \equiv H+i A \sqrt{\frac{1-\tau}{1+\tau}}$ and $H$ and $A$ are hermitian matrices with equal Gaussian weight. The parameter $\tau$ measures the degree of non-hermiticity, a concept which was introduced in 40. In particular this allows to take 


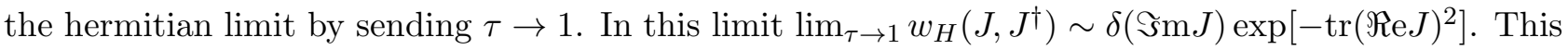
possibility makes the difference between the complex matrix model and two-matrix models particularly transparent. In the former we have introduced polynomials of a complex variable $P_{j}(z)$ as well as their complex conjugate. While we can smoothly take the limit of $z$ becoming real here, this is not possible in the two-matrix model solved by the method of bi-orthogonal polynomials. The orthogonal polynomials for the weight in eq. (4.6) can be shown to be Hermite polynomials [39],

$$
\tilde{P}_{j}(z)=\left(\frac{\tau}{2}\right)^{\frac{j}{2}} H_{j}\left(\frac{z}{\sqrt{2 \tau}}\right), h_{j}=\pi j ! \sqrt{1-\tau^{2}} .
$$

Using that the Hermite polynomials have real coefficients their kernel is given by

$$
\kappa_{N}(z, \bar{u})=\frac{1}{\pi \sqrt{1-\tau^{2}}} \sum_{j=0}^{N-1} \frac{1}{j !}\left(\frac{\tau}{2}\right)^{j} H_{j}\left(\frac{z}{\sqrt{2 \tau}}\right) H_{j}\left(\frac{\bar{u}}{\sqrt{2 \tau}}\right) .
$$

An other example is for a bounded domain $D$ in the complex plane, namely the disk $D_{r}=\{z \in \mathbb{C}$ : $|z|<r\}$ or the circle $C_{r}=\{z \in \mathbb{C}:|z|=r\}$. In both cases the orthogonal polynomials with respect to the flat measure $w(z, \bar{z})=1$ are again given by monic powers but with different normalization coefficients,

$$
\begin{array}{ll}
\tilde{P}_{j}(z)=z^{j}, & h_{j}=\frac{\pi}{j+1} r^{2 j+2}, \quad \text { in } D_{r}, \\
\tilde{P}_{j}(z)=z^{j}, & h_{j}=2 \pi r^{2 j+1}, \quad \text { in } C_{r},
\end{array}
$$

and the bare kernels read

$$
\begin{aligned}
& \kappa_{N}(v, \bar{u})=\frac{1}{\pi r^{2}} \sum_{j=0}^{N-1}(j+1)\left(\frac{v \bar{u}}{r^{2}}\right)^{j}=\frac{1}{\pi r^{2}} \frac{1-a^{N}(1+N-a N)}{(1-a)^{2}}, \quad \text { in } D_{r}, \\
& \kappa_{N}(v, \bar{u}) \quad=\frac{1}{2 \pi r} \sum_{j=0}^{N-1}\left(\frac{v \bar{u}}{r^{2}}\right)^{j}=\frac{1}{2 \pi r} \frac{1-a^{N}}{(1-a)}, \quad \text { in } C_{r} \quad, \quad a=\frac{v \bar{u}}{r^{2}} .
\end{aligned}
$$

Actually, all domains and weight functions in the complex plane which are invariant under the rotation $z \rightarrow e^{i \theta} z, \forall \theta \in[0,2 \pi]$ (i.e. they are function only of the radial coordinate $\rho=|z|$ ), give orthogonal polynomials which are monic powers, i.e. $\tilde{P}_{j}(z)=z^{j}$ with normalization coefficients given by

$$
h_{j}=2 \pi \int_{0}^{r} d \rho \rho^{2 j+1} w(\rho, \rho) .
$$

The Gaussian case $w_{G}(z, \bar{z})$ we considered above belongs to this class, and therefore the same arguments apply here. In particular, eq. (4.4) holds exactly for all the elements of this class, since it is independent of the particular expression for $h_{j}$.

A more interesting case is for a bounded domain $D$ which is not rotationally invariant, e.g. the ellipse

$$
D_{r}^{e l l}=\left\{z \in \mathbb{C}:|z|<\frac{1}{2}\left(r e^{i \theta}+c^{2} r^{-1} e^{-i \theta}\right), \forall \theta \in[0,2 \pi]\right\}, \quad r \geq c \geq 0
$$

or its boundary $C_{r}^{e l l}=\partial D_{r}^{e l l}$. The shape of the ellipse is parameterized by two parameters, $r$ and the semi-focal length $c$. Obviously the semi-minor/major axis lengths are equal to $r-c^{2} r^{-1}, r+c^{2} r^{-1}$, 
respectively. It is easy to see that in this case the monic orthogonal polynomials with respect to a flat measure $w(z, \bar{z})=1$, are given by the Chebyshev polynomials of the second kind:

$$
\tilde{P}_{j}(z)=\left(\frac{c}{2}\right)^{j} U_{j}\left(\frac{z}{c}\right), \quad h_{j}=\frac{\pi}{2^{2 j+2}(j+1)}\left(r^{2 j+2}-\left(\frac{c^{2}}{r}\right)^{2 j+2}\right) .
$$

It is easy to check that eq. (4.13) reduces to eq. (4.8) in the limit $c \rightarrow 0$. The kernel reads:

$$
\kappa_{N}(v, \bar{u})=\frac{4}{\pi} \sum_{j=0}^{N-1} \frac{c^{2 j}(j+1)}{r^{2 j+2}-\left(c^{2} r^{-1}\right)^{2 j+2}} U_{j}\left(\frac{v}{c}\right) U_{j}\left(\frac{\bar{u}}{c}\right) .
$$

The final example we want to give are the generalized Laguerre polynomials in the complex plane as recently introduced in [29]. For this chiral complex eigenvalue model no matrix representation is known so far and we have to slightly modify our definitions (2.4) and (2.8). The partition function is given by

$$
\mathcal{Z}_{N}^{c h} \equiv \int_{D} \prod_{i=1}^{N}\left(d^{2} z_{i} w_{c h}\left(z_{i}, \bar{z}_{i}\right)\right)\left|\Delta_{N}\left(z^{2}\right)\right|^{2},
$$

with $D$ being the full complex plane and a weight

$$
w_{c h}(z, \bar{z}) \equiv|z|^{2 a+1} \exp \left[-\frac{1}{1-\tau^{2}}\left(|z|^{2}-\frac{\tau}{2}\left(z^{2}+\bar{z}^{2}\right)\right)\right], \quad a>-\frac{1}{2} \in \mathbb{R} .
$$

We could also allow for more general weight functions and domain $D$, the crucial point for the model to be chiral being the squared argument inside the Vandermonde determinant. The orthogonal polynomials are then necessarily polynomials in $z^{2}$ :

$$
h_{k} \delta_{k l}=\int_{D} d^{2} z w(z, \bar{z}) \tilde{P}_{k}^{c h}\left(z^{2}\right) \overline{\tilde{P}_{l}^{c h}\left(z^{2}\right)} .
$$

The characteristic polynomials consequently have to be defined in terms of the eigenvalues

$$
\left\langle\prod_{i=1}^{N}\left(\prod_{j=1}^{K}\left(v_{j}^{2}-z_{i}^{2}\right) \prod_{l=1}^{L}\left(\bar{u}_{l}^{2}-\bar{z}_{i}^{2}\right)\right)\right\rangle_{N} .
$$

The whole proof of Theorem (2.11) goes through as before, replacing all arguments by their square on the right hand side.

The finite- $N$ orthogonal polynomials for our example eq. (4.16) are given by the generalized Laguerre polynomials [31]

$$
\tilde{P}_{k}^{c h}\left(z^{2}\right)=(-1)^{k} \frac{\Gamma(a+1) k !}{\Gamma(a+k+1)} L_{k}^{a}\left(\frac{z^{2}}{2 \tau}\right) .
$$

Their norm can be calculated to be

$$
\begin{aligned}
h_{k} & =\frac{f^{a}(\tau) \Gamma(a+1) k !}{\tau^{2 k} \Gamma(a+k+1)} \\
f^{a}(\tau) & =\int_{D} d^{2} z w_{c h}(z, \bar{z})=\pi \Gamma\left(a+\frac{3}{2}\right)\left(1-\tau^{2}\right)^{\frac{a}{2}+\frac{3}{4}} \mathcal{P}_{a+\frac{1}{2}}\left(\frac{1}{\sqrt{1-\tau^{2}}}\right),
\end{aligned}
$$

where $\mathcal{P}_{a+\frac{1}{2}}(x)$ is the Legendre function. The bare kernel is thus reading

$$
\kappa_{N}(z, \bar{u})=\frac{1}{f^{a}(\tau)} \sum_{k=0}^{N-1} \frac{\Gamma(a+1) k !}{\Gamma(a+k+1)} \tau^{2 k} L_{k}^{a}\left(\frac{z^{2}}{2 \tau}\right) L_{k}^{a}\left(\frac{\bar{u}^{2}}{2 \tau}\right) .
$$




\subsection{A duality relation}

In this subsection we wish to prove a duality relation that relates products of characteristic polynomials of different (finite) matrix size. This generalizes the relation found in [11, 12] to complex matrices. The proof goes back to a relation between determinants of Hermite polynomials [11] which we will use. Therefore our duality relation will only hold for the weight eq. (4.5) having Hermite polynomials in the complex plane.

To begin with we consider Theorem eq. (2.11) at $L=0$ where only polynomials and no kernels appear. Next we take the limit of equal arguments $v_{i}=v, \forall i=1, \ldots, K$. We obtain

$$
\left\langle\operatorname{det}[v-J]^{K}\right\rangle_{N}=\frac{1}{\prod_{j=1}^{K-1} j !} \operatorname{det}_{1 \leq l, m \leq K}\left[\partial_{v}^{(l-1)} \tilde{P}_{N+m-1}(v)\right] .
$$

This looks exactly as for the hermitian matrix model (e.g. in [11]). Next we insert the orthonormalized Hermite polynomials eq. (4.6) for the chosen weight eq. (4.5) and obtain

$$
\begin{aligned}
\left\langle\operatorname{det}[v-J]^{K}\right\rangle_{N} & =\frac{1}{\prod_{j=1}^{K-1} j !}\left(\frac{\sqrt{2 \tau}}{2}\right)^{N K+K(K-1) / 2} \sqrt{2 \tau}-K(K-1) / 2 \operatorname{det}_{1 \leq l, m \leq K}\left[H_{N+m-1}^{(l-1)}\left(\frac{v}{\sqrt{2 \tau}}\right)\right] \\
& =\frac{1}{\prod_{j=1}^{K-1} j !}\left(\frac{\sqrt{2 \tau}}{2}\right)^{N K}\left(\frac{-1}{2}\right)^{K(K-1) / 2} \operatorname{det}_{1 \leq l, m \leq K}\left[H_{N+m+l-2}\left(\frac{v}{\sqrt{2 \tau}}\right)\right] .
\end{aligned}
$$

In the first step we have taken out all factors of the determinant stemming from the monic normalization and the differentiation, where $H_{N+m-1}^{(l-1)}(v / \sqrt{2 \tau})$ denotes the derivative with respect to the full argument $v / \sqrt{2 \tau}$. In the second step we have used the following relation for the Hermite polynomials,

$$
H_{N}^{\prime}(x)=2 x H_{N}(x)-H_{N+1}(x),
$$

which holds algebraically for any $x$ being real or complex. We have thus shifted the derivative into the index of the polynomials, taking out all signs. We can now use the following relation among determinants of Hermite polynomials as being shown in [11]

$$
\frac{\operatorname{det}_{1 \leq l, m \leq K}\left[H_{N+m+l-2}(x)\right]}{(-2)^{K(K-1) / 2} \prod_{j=1}^{K-1} j !}=(-i)^{K N} \frac{\operatorname{det}_{1 \leq l, m \leq N}\left[H_{K+m+l-2}(i x)\right]}{(-2)^{N(N-1) / 2} \prod_{j=1}^{N-1} j !} .
$$

This relation was proved in [11] for real $x$ only. However, the relation is purely algebraic as it relates two polynomials after multiplying out the two determinants. We can therefore also allow for $x$ to be complex, in particular $x=v / \sqrt{2 \tau}$. Note also the factor of $i$ in the argument on the right hand side.

Replacing the determinant of size $K \times K$ in eq. (4.23) by a determinant of size $N \times N$ with the help of eq. (4.25), we arrive at the following remarkable identity:

$$
\left\langle\operatorname{det}[v-J]^{K}\right\rangle_{N}=(-i)^{K N}\left\langle\operatorname{det}[i v-J]^{N}\right\rangle_{K}
$$

interchanging the power of the product with the matrix size. It is identical to the corresponding equation in [11] replacing the complex matrix $J$ and argument $v$ by the respective hermitian matrix $H$ and real argument $x$. The normalization in eq. (4.26) can be easily checked taking the limit $v \rightarrow \infty$. Another peculiar case is $N=K$ at $v=0$. Still, the duality relation is satisfied as both expectation values which are now equal vanish for odd $N=K$ due to symmetry.

\footnotetext{
${ }^{6}$ In [1] this relation was shown for the determinant of $C_{n}(x) \equiv 2^{-n} H_{n}(x)$, but the factors of 2 can be easily taken out. We further note a typo in eq. (3.16) there.
} 
The relation eq. (4.26) trivially extends to the Ginibre ensemble eq. (4.1), which can be obtained by taking $\tau \rightarrow 0$ on the Hermite polynomials eq. (4.6). It is because in the Ginibre case all expectation values of powers of $J$ vanish. The same argument applies for all weights with spherical symmetry and monomials as orthogonal polynomials, as in eqs. (4.8) and (4.9). We suspect that the duality also holds for other weight functions.

\section{The microscopic large- $N$ limit and universality}

The explicit examples given in the last Section are a good starting point for the analysis of the asymptotic large- $N$ limit. To this aim we first rescale the complex eigenvalues by $z_{i} \rightarrow \sqrt{N} z_{i}$ in order to have $N$ explicitly in the exponent of the weight functions eqs. (4.5) or (4.16), to which we will restrict in this Section. Then we focus on the microscopic large- $N$ limit where the correlations of eigenvalues at small distance $\mathrm{O}\left(\left|z_{i}-z_{j}\right|\right) \ll 1$ are considered. For spectral correlators at a distance of $\mathrm{O}(1)$ (the so-called macroscopic limit) we refer to [22, 26].

Furthermore, one has to distinguish two different ways of taking the microscopic $N \rightarrow \infty$ limit [40]. Starting from a general weight such as in eq. (2.6) or simply from the Ginibre ensemble eq. (4.1) we will always end up in a regime which is called strong non-hermiticity. Introducing an additional parameter $\tau \in[0,1]$ as in eq. (4.5) will allow us to reach also the regime of weak non-hermiticity, when taking simultaneously $N \rightarrow \infty$ and $(1-\tau) \rightarrow 0$ while keeping the product fixed. The latter limit introduced in 40] permits to smoothly interpolate between correlations of real eigenvalues on one hand and complex eigenvalues in the strong non-hermiticity limit on the other hand.

Let us begin with the weak non-hermiticity limit. Here we focus on a weight function of the form of eq. (2.6), which is in terms of eigenvalues

$$
w(z, \bar{z}) \equiv \exp \left[-N\left(\frac{1}{1-\tau^{2}}\left(|z|^{2}-\frac{\tau}{2}\left(z^{2}+\bar{z}^{2}\right)\right)+\frac{1}{2} \sum_{k=2}^{d} \frac{g_{2 k}}{2 k}\left(z^{2 k}+\bar{z}^{2 k}\right)\right)\right],
$$

which generalizes the weight eq. (4.5)]. Here the $g_{2 k} \in \mathbb{R}$ are real coupling constants and $\tau \in[0,1]$ measures the degree of non-hermiticity as explained after eq. (4.5). The weak non-hermiticity limit is defined by taking [40]

$$
\lim _{\substack{N \rightarrow \infty \\ \tau \rightarrow 1}} N\left(1-\tau^{2}\right) \equiv \alpha^{2}
$$

to be constant. At the same time we rescale the complex eigenvalues according to

$$
N z=N(\Re \mathrm{e} z+i \Im \mathrm{m} z) \equiv \xi,
$$

which is the microscopic limit at the origin since $z \rightarrow 0$ while $N \rightarrow \infty$. It is important to note that the scaling is done with the same power of $N$ as for real eigenvalues, in contrast to the strong non-hermiticity limit (see eq. (5.10) below). This has important consequences on the asymptotic limit of the orthogonal polynomials as well.

We will now give the asymptotic results for the orthonormal polynomials of eq. (5.1) and their kernel as they were derived in [31]:

$$
\lim _{\substack{n, N \rightarrow \infty \\
\tau \rightarrow 1}} P_{n}\left(z=\frac{\xi}{N}\right)=\left[\sqrt{2} N u(t)^{\prime} \alpha^{-1} \pi^{-\frac{3}{2}}\right]^{\frac{1}{2}} \mathrm{e}^{-\frac{\alpha^{2}}{4} u(t)^{2}}\left\{\begin{array}{ll}
\cos (u(t) \xi) & n \text { even } \\
\sin (u(t) \xi) & n \text { odd }
\end{array}, t=\frac{n}{N} .\right.
$$

\footnotetext{
${ }^{7}$ Strictly speaking the integral over the weight function eq. (5.1) cannot be made convergent for any choice of signs of the coupling constants $g_{2 k}$. Consequently one would either have to restrict the domain $D$ to be compact or to introduce a small term $\sim \epsilon|z|^{2 d+2}$ to achieve convergence. However, in the weakly non-hermitian large- $N$ limit the support of the eigenvalues does not only become compact but also gets projected onto the real line. The correlators depend on complex variables only microscopically. For that reason we ignore the aforementioned technical subtleties.
} 
In [31] the full kernel eq. 2.13) including the weights was given. Using the fact that the asymptotic limit of the weight function reads

$$
w(z, \bar{z}) \rightarrow \exp \left[-\frac{2}{\alpha^{2}}(\Im \mathrm{m} z)^{2}\right]
$$

we obtain for $\kappa_{N}\left(z_{1}, \overline{z_{2}}\right)$ appearing in Theorem (2.11)

$$
\lim _{N \rightarrow \infty} \frac{1}{N^{2}} \kappa_{N}\left(z_{1}=\frac{\xi_{1}}{N}, \overline{z_{2}}=\frac{\bar{\xi}_{2}}{N}\right)=\frac{2}{\alpha \pi} \int_{0}^{\pi \rho_{1}(0)} \frac{d u}{\sqrt{2 \pi}} \mathrm{e}^{-\frac{\alpha^{2}}{2} u^{2}} \cos \left[u\left(\xi_{1}-\bar{\xi}_{2}\right)\right] .
$$

Here we have introduced the spectral density of real eigenvalues $\rho_{t}(x)$ corresponding to the hermitian limit of the potential in eq. (5.1):

$$
\begin{aligned}
\rho_{t}(x) & \equiv \frac{1}{2 \pi} \sum_{j=1}^{m} \frac{g_{2 j}}{t} \sum_{k=0}^{j-1}\left(\begin{array}{c}
2 k \\
k
\end{array}\right) r(t)^{k} x^{2(j-k-1)} \sqrt{4 r(t)-x^{2}}, \\
1 & =\frac{1}{2} \sum_{k=1}^{m} \frac{g_{2 k}}{t}\left(\begin{array}{c}
2 k \\
k
\end{array}\right) r(t)^{k} .
\end{aligned}
$$

The function $u(t)$ in eq. (5.4) is related to the spectral density by

$$
\pi t \rho_{t}(0) \equiv u(t)
$$

Finally the asymptotic of the norm of the polynomials is:

$$
h_{N} \rightarrow \sqrt{\frac{u^{\prime}(t)}{\alpha}}
$$

The remaining $N$-dependence of the norm $h_{N} \sim N$ ! in the prefactors of eq. (2.11) has to be canceled by a suitable normalization. Inside the determinant it is needed when passing from the monic to the orthonormal polynomials to make the asymptotic limit of the latter well defined. To summarize we have determined the weakly non-hermitian large- $N$ limit of the characteristic polynomials as stated in Theorem eq. (2.11), when inserting eqs. (5.4), (5.6) and (5.9). Hence it is also universal.

In [40] the weak non-hermiticity limit away from the origin was also analyzed, for the Gaussian weight of eq. (4.5). The corresponding kernel is exactly of the same form of eq. (5.6), with replacing $\pi \rho_{1}(0) \rightarrow \sqrt{1-X^{2} / 4}$ which is the Wigner semicircle density at the point of investigation $X \in \mathbb{R}$. While the norms $h_{N}$ still behave as in eq. (5.9) with $u(t)=\sqrt{2 t}$ the asymptotic polynomials will acquire an additional oscillating phase $\sim \cos (\sqrt{2 t}(\xi+N X))$ as we now microscopically rescale the deviation from point $X: z=X+\xi / N$, with $z, \xi \in \mathbb{C}$. In other words their asymptotic limit does no longer exist. Therefore only for $K=L$ a smooth limit is found at weak non-hermiticity away from the origin (a similar situation is found below at strong non-hermiticity). Furthermore the question of universality away from the origin remains open.

We now turn to the microscopic limit at strong non-hermiticity where we keep $\tau \in[0,1)$ fixed while $N \rightarrow \infty$. The microscopic origin scaling limit is defined here by letting $z \rightarrow 0$ and $N \rightarrow \infty$ keeping

$$
\sqrt{N} z=\sqrt{N}(\Re \mathrm{e} z+i \Im \mathrm{m} z) \equiv \xi,
$$

constant. The reason for the different scaling is that now the eigenvalues will become dense on a compact domain which truly extends into the complex plane. This has an immediate consequence as the asymptotic large- $N$ of the orthogonal polynomials no longer exists. This can be seen in the 
simplest example of the Ginibre ensemble eq. (4.1) or of the bounded domains eq. (4.8), (4.9). The polynomials $P_{N}(z)=z^{N} / N$ ! from eq. (4.2) will certainly no longer have a smooth large- $N$ expansion. The same problem occurs for the Hermite polynomials in the complex plane. Expanding for example

the even powers, $H_{2 j}\left(z \sqrt{\frac{N}{2 \tau}}\right) \sim \cos \left[\sqrt{4 j+1} z \sqrt{\frac{N}{2 \tau}}\right]$ it can be seen that while the weak scaling limit eq. (5.3) is smooth (keeping $j / N=t$ fixed) the strong limit from eq. (5.10) does not exist. While on the real line the existence of an asymptotic expansion of the polynomials and the kernel are directly related through the Christoffel-Darboux formula eq. (2.18) this is no longer the case here, since such a relation does not hold in general in the complex plane. However the asymptotic limit of the kernel eq. (4.3) still exist and it is given by the exponential function being smooth. The result for the weight in eq. (4.5) reads after splitting of the weight factors [40]:

$$
\lim _{N \rightarrow \infty} \frac{1}{N} \kappa_{N}\left(z_{1}=\frac{\xi_{1}}{\sqrt{N}}, \bar{z}_{2}=\frac{\bar{\xi}_{2}}{\sqrt{N}}\right)=\frac{1}{\pi\left(1-\tau^{2}\right)} \exp \left[\frac{1}{\left(1-\tau^{2}\right)}\left(\xi_{1} \bar{\xi}_{2}-\frac{\tau}{2}\left(\xi_{1}^{2}+\bar{\xi}_{2}^{2}\right)\right)\right] .
$$

For $\tau \rightarrow 0$ we recover the exponential Ginibre kernel [25]. Taking into account the rescaled norms $h_{N} \sim \sqrt{1-\tau^{2}}$ we can read off the asymptotic strongly non-hermitian large- $N$ limit of our characteristic polynomials eq. (2.11) for $K=L$. The question of universality of eq. (5.11) for more general weight functions remains an open question. This has to be compared with the situation in the macroscopic large- $N$ limit. There, it has been recently shown that the two-point resolvent is universal but the three- and four-point function are not [22].

The same analysis for the microscopic large- $N$ limit at weak and strong non-hermiticity can be done for the chiral ensemble of complex eigenvalues introduced in eqs. (4.15) and (4.16). The asymptotic expressions for the norms, polynomials and kernel in the weak limit, and the norms and kernel in the strong limit have already been explicitly given in [31] and we will not repeat them here. We find the same phenomenon as above that only the case $K=L$ at strong non-hermiticity makes sense asymptotically. At weak non-hermiticity there are no such restrictions. The issue of universality for the chiral ensemble remains an open problem.

We finish this Section with a remark: in the mathematical literature the large- $N$ macroscopic limit of our kernel $K_{N}(v, \bar{u})$ is known as Bergman kernel function or Szegö kernel function, according to the case where the weight function is defined on a domain in the complex plane or on its boundary. Both these kernels are objects of fundamental importance in the theory of conformal mapping [32].

\section{The BMN large- $N$ limit}

In this Section we apply the results of Sections 3 and 4 to the study of objects like $\left\langle\operatorname{tr} J^{K} \operatorname{tr} J^{\dagger K}\right\rangle_{N}$ in the limit where both $N$ and $K$ become large. Such quantities have become of interest in the correspondence between pp-wave strings and supersymmetric Yang-Mills theory [23]. In this case the expectation values are evaluated with respect to the weight of the Ginibre ensemble eq. (4.1). It has been found that besides the usual (macroscopic) large- $N$ limit, one can consider also $K, N \rightarrow \infty$ such that $\frac{K^{2}}{N}$ is finite. This limit is called BMN limit after [24]. In a sense in this limit the expectation value becomes part of the matrix model action and the weight is no longer truly Gaussian as in eq. (4.1). It would be very interesting to study more general insertions to the matrix model action and to see if such models permit a large- $N$ BMN limit. In this Section we will study the weight eq. (4.5) for the Hermite polynomials as an example.

It is convenient to introduce products of resolvent operators such as

$$
W(z, \bar{u}) \equiv\left\langle\operatorname{tr} \frac{1}{z-J} \operatorname{tr} \frac{1}{\bar{u}-J^{\dagger}}\right\rangle=\frac{1}{z \bar{u}} \sum_{l, m=0}^{\infty} \frac{1}{z^{l} \bar{u}^{m}}\left\langle\operatorname{tr} J^{l} \operatorname{tr} J^{\dagger m}\right\rangle
$$


in order to generate expectation values of product of traces of powers of matrices. However, at finite- $N$ only few closed formulas for a small number of traces are known so far, such as 23]

$$
\left\langle\operatorname{tr} J^{l} \operatorname{tr} J^{\dagger m}\right\rangle=\delta_{l m}\left(\frac{\Gamma(N+l+1)}{\Gamma(N)}-\frac{\Gamma(N+1)}{\Gamma(N-l)}\right)
$$

for the Ginibre weight eq. (4.1). More general multi-point resolvents are known only in the asymptotic large- $N$ limit so far [26].

The products of characteristic polynomials we have calculated in eq. (2.11) are generating functions that may be used to extract information about the spectrum. In fact they generate all the elementary symmetric functions $c_{r}$ of the eigenvalues:

$$
\begin{aligned}
\operatorname{det}[v+J] & =v^{N} \sum_{r=0}^{N} c_{r} v^{-r}, \\
c_{r} & \equiv \sum_{i_{1}<\ldots<i_{r}}^{N} z_{i_{1}} \cdots z_{i_{r}}, \quad r=1, \ldots, N .
\end{aligned}
$$

Similarly we have $\operatorname{det}\left[\bar{u}+J^{\dagger}\right]=\bar{u}^{N} \sum_{r=0}^{N} \bar{c}_{r} \bar{u}^{-r}$ for the hermitian conjugate. The elementary symmetric functions $c_{r}$ can be expressed by the power sums

$$
p_{r} \equiv \sum_{i=1}^{N} z_{i}^{r}=\operatorname{tr} J^{r}, \quad r=1, \ldots, N
$$

Namely, the two sets are related by

$$
c_{r}=\frac{1}{r !} \operatorname{det}_{1 \leq i, j, \leq r}\left[S_{i j}\right], \quad S_{i j}=\left\{\begin{array}{cl}
p_{j-i+1} & \text { if } j \geq i \\
j & \text { if } j=i-1 \\
0 & \text { if } j<i
\end{array} .\right.
$$

As a first example we can apply the Theorem eq. (2.11) for $K=L$ using the finite- $N$ result eq. (4.3) for the Gaussian weight:

$$
\begin{aligned}
\left\langle\operatorname{det}[v-J] \operatorname{det}\left[\bar{u}-J^{\dagger}\right]\right\rangle_{N} & =h_{N} \kappa_{N+1}(v, \bar{u})=N ! \sum_{j=0}^{N} \frac{1}{j !}(v \bar{u})^{j} \\
& =\sum_{l, m=0}^{N}\left\langle c_{l} \bar{c}_{m}\right\rangle_{N}(-v)^{N-l}(-\bar{u})^{N-m} .
\end{aligned}
$$

Comparing coefficients we obtain

$$
\left\langle c_{l} \bar{c}_{m}\right\rangle_{N}=\delta_{l m} \frac{N !}{(N-l) !}
$$

In the large- $N$ limit it yields

$$
\begin{aligned}
\left\langle c_{l} \bar{c}_{l}\right\rangle_{N} & =N^{l}\left(1-\frac{l(l-1)}{2 N}+\frac{1}{12}\left(\begin{array}{l}
l \\
2
\end{array}\right)\left(3(l-1)^{2}-l-1\right) \frac{1}{N^{2}}+\ldots\right) \\
& =N^{l}\left(1+\sum_{h=1}^{\infty} a_{h} \frac{l^{2 h}}{N^{h}}+\ldots\right)
\end{aligned}
$$


and consequently an expansion in $l^{2} / N$ exists. We note that the expansion goes in powers of $\frac{1}{N}$ instead of the usual genus expansion in powers of $\frac{1}{N^{2}}$. This is due to the fact that eq. (6.7) contains expectation values of all combinations $\left\langle p_{i_{1}} \cdots p_{i_{r}} \bar{p}_{j_{1}} \cdots \bar{p}_{j_{r}}\right\rangle_{N}$ with $\sum_{s=1}^{r} i_{s}=\sum_{s=1}^{r} j_{s}=r$. So far such expectation values were not known explicitly for finite- $N$ in a closed form. If we could manage to disentangle all expectation values of $p_{r}$ and $\bar{p}_{r}$ alone, the individual terms would all have a genus expansion in $\frac{1}{N^{2}}$, with different prefactors in $N$ however. The fact that the BMN limit of eq. (6.7) exists is quite remarkable. This holds for the Ginibre ensemble. We wish to mention however, that for a more general weight function the expansion is generically in powers of $\frac{1}{N}$. For example the next to leading order of the free energy in $\frac{1}{N}$ can be calculated [22].

The difficulty to extract formulas like eq. (6.2) from eq. (6.7) is that each elementary symmetric functions $c_{r}$ depends on all lower powers $p_{1}, \ldots, p_{r}$ through the determinant in eq. (6.5). The fact that the $c_{r}$ can be constructed recursively, $n c_{n}=\sum_{r=1}^{n}(-1)^{r-1} p_{r} c_{n-r}$, is also not enough to extract each single term. We have therefore not been able to derive new finite- $N$ expectation values of traces alone, that would generalize eq. (6.2). However, we have explicitly checked in few cases that eq. (6.7) leads to eq. (6.2).

We will now address the question whether the existence of the BMN limit is a generic property of complex matrix models or if it is specific to the Ginibre ensemble. Therefore we look at the ensemble with weight eq. (4.5) of Hermite polynomials. In this model single powers of traces $\left\langle\operatorname{tr} J^{l}\right\rangle$ do not vanish any longer for $\tau \neq 0$. The simplest nontrivial characteristic polynomial is given by

$$
\langle\operatorname{det}[v-J]\rangle_{N}=\tilde{P}_{N}(v)=\left(\frac{\tau}{2}\right)^{\frac{N}{2}} H_{N}\left(\frac{v}{\sqrt{2 \tau}}\right),
$$

where we have used eq. (4.6). In order to read off the coefficients from eq. (6.3) by expanding the right hand side we distinguish between even and odd $N$. For $N=2 n$ we obtain

$$
\begin{aligned}
\langle\operatorname{det}[v-J]\rangle_{2 n} & =\sum_{l=0}^{n}\left\langle c_{2 n-2 l}\right\rangle_{2 n}(-v)^{2 l} \\
& =\sum_{l=0}^{n}(-1)^{n+l}(2 \tau)^{n-l}\left(\begin{array}{l}
n \\
l
\end{array}\right) \frac{\Gamma\left(n+\frac{1}{2}\right)}{\Gamma\left(l+\frac{1}{2}\right)} v^{2 l},
\end{aligned}
$$

where we have used the fact that our weight is even. Repeating the same analysis for $N=2 n+1$ and using an identity for the Gamma function we arrive at

$$
\left\langle c_{2 l}\right\rangle_{N}=\left(-\frac{\tau}{2}\right)^{l} \frac{N !}{l !(N-2 l) !}, \quad\left\langle c_{2 l+1}\right\rangle_{N}=0,
$$

which holds for any $N$. Looking at eq. (6.7) we can see that again the limit $l, N \rightarrow \infty$ with $l^{2} / N$ fixed exists. Such a large- $N$ limit thus seems to be a generic property of complex matrix models. We note however, that the expectation value eq. (6.11) has to be properly normalized since both $\tau^{l}$ and $1 / l$ ! vanish for $0<\tau<1$ at large- $l$.

\section{Conclusions}

We have studied products of characteristic polynomials of complex matrices and their hermitian conjugate. For hermitian matrix models several equivalent determinant formulas hold for characteristic polynomials. They contain orthogonal polynomials and their kernel, which are related through the Christoffel-Darboux formula. For complex matrices this is no longer the case. The number of characteristic polynomials and their conjugate completely fixes the result which in general is a determinant 
of both the orthogonal polynomials in the complex plane and their respective kernel. If we only consider characteristic polynomials of complex matrices without their conjugate our result reduces to a determinant over polynomials only, which is identical to the result of the hermitian matrix model. This does not come as a surprise since our proof only uses the existence of orthogonal polynomials in the complex plane and holds despite of the breakdown of the three-step recursion relation and the Christoffel-Darboux identity.

Next we gave an interpretation of our results in terms of orthogonal polynomials in the complex plane, with weights including determinants, and their corresponding kernels. We have provided explicit examples for complex polynomials at finite- $N$ such as Hermite, Laguerre or Chebyshev polynomials in the complex plane. From them we have constructed orthogonal polynomials for a class of more general weights.

At finite- $N$ we proved a duality relation for the weight with Hermite polynomials in the complex plane. It relates expectation values of different powers of characteristic polynomials with respect to a different matrix size, generalizing a known duality for the Gaussian Hermitian matrix model.

We have also studied the microscopic large- $N$ limit of characteristic polynomials when the arguments are rescaled with the matrix size $N$. In the weak non-hermiticity limit all products of characteristic polynomials are universal for a large class of weight functions. The strongly non-hermitian large- $N$ limit is smooth only for products with an equal number of characteristic polynomials and their hermitian conjugate. This is again a peculiar implication of the breakdown of the Christoffel-Darboux identity. Although in general the orthogonal polynomials do no longer have a smooth asymptotic limit, their kernel still can and does have a smooth limit. The issue of universality of the large- $N$ kernel at strong non-hermiticity remains open at present.

Furthermore we have argued that the existence of the BMN large- $N$ limit is a generic phenomenon for complex matrix models. In using the fact that characteristic polynomials generate the elementary symmetric functions we have shown that for quite general expectation values such a limit exists. In principle from our finite- $N$ results one can compute iteratively expectation values of single powers of traces alone. However it would be more useful to find a closed expression for that, if any.

It would be also very interesting to extend our analysis to include negative powers of moments in the expectation values. We suspect that a result similar to that of hermitian matrices holds, where the Cauchy transformation of the orthogonal polynomials occurs. This would provide us with an alternative way to evaluate complex eigenvalue correlations at finite- $N$ and infinite- $N$.

Acknowledgments: We wish to thank A. D'Adda, F. David and M.L. Mehta for useful discussions and P. Forrester for correspondence. This work was supported by the European network on "Discrete Random Geometries" HPRN-CT-1999-00161 EUROGRID and by a Heisenberg fellowship of the Deutsche Forschungsgemeinschaft.

\section{References}

[1] A.V. Andreev and B.D. Simons, Phys. Rev. Lett. 75 (1995) 2304.

[2] E.V. Shuryak and J.J.M. Verbaarschot, Nucl. Phys. A560 (1993) 306 hep-th/9212088; J. Verbaarschot, Phys. Rev. Lett. 72 (1994) 2531 hep-th/9401059.

[3] J.P. Keating and N. Snaith, Commun. Math. Phys. 214 (2000) 57.

[4] T. Guhr, A. Müller-Groeling and H.A. Weidenmüller, Phys. Rep. 299 (1998) 190 condmat/9707301. 
[5] Y.V. Fyodorov, Nucl. Phys. B621 (2002) 643 math-ph/0106006; Y.V. Fyodorov and E. Strahov, Nucl. Phys. B630 (2002) 453 math-ph/0201045.

[6] E. Kanzieper, Replica field theories, Painleve transcendents and exact correlation functions, condmat/0207745.

[7] K. Splittorff and J.J.M. Verbaarschot, Replica Limit of the Toda Lattice Equation, condmat/0209594.

[8] Y.V. Fyodorov and E. Strahov, On correlation functions of characteristic polynomials for chiral Gaussian Unitary Ensemble hep-th/0205215; Y.V. Fyodorov and G. Akemann, Comment on the article cond-mat/0209594 by K. Splittorff and J.J.M. Verbaarschot cond-mat/0210647.

[9] Y.V. Fyodorov and E. Strahov, An exact formula for general spectral correlation function of random Hermitian matrices, math-ph/0204051.

[10] E. Strahov and Y.V. Fyodorov, Universal Results for Correlations of Characteristic Polynomials: Riemann-Hilbert Approach, math-ph/0210010.

[11] M.L. Mehta and J.-M. Normand, J. Phys. A: Math. Gen. 34 (2001) 1 cond-mat/0101469.

[12] P.J. Forrester and N.S. Witte, Commun. Math. Phys. 219 (2001) 357 math-ph/0103025].

[13] P.H. Damgaard, Phys. Lett. B424 (1998) 322 [hep-th/9711110]; G. Akemann and P.H. Damgaard, Nucl. Phys. B528 (1998) 411 [hep-th/9801133; Phys. Lett. B432 (1998) 390 hep-th/9802174.

[14] P.H. Damgaard and S.M. Nishigaki, Phys. Rev. D57 (1998) 5299-5302 hep-th/9711096.

[15] T. Wilke, T. Guhr, and T. Wettig, Phys. Rev. D57, 6486 (1998) hep-th/9711057.

[16] P.H. Damgaard, Proceedings of "Quantum Chromodynamics 98", Paris 1998, p. 296-303 [hepth/9807026].

[17] T. Nagao and S.M. Nishigaki, Phys. Rev. D62 (2000) 065006 hep-th/0001137.

[18] G. Akemann and E. Kanzieper, Phys. Rev. Lett. 85 (2000) 1174 [hep-th/0001188].

[19] T. Nagao and S.M. Nishigaki, Phys. Rev. D63 (2001) 045011 hep-th/0005077.

[20] E. Brézin and S. Hikami, Commun. Math. Phys. 214 (2000) 111 math-ph/9910005; Commun. Math. Phys. 223 (2001) 363 math-ph/0103012.

[21] O. Agam, E. Bettelheim, P. Wiegmann and A. Zabrodin, Phys. Rev. Lett. 88 (2002) 236802 cond-mat/0111333.

[22] P. Wiegmann and A. Zabrodin, Large scale correlations in normal and general non-Hermitian matrix ensembles, hep-th/0210159; A. Zabrodin, New applications of non-hermitian random matrices, cond-mat/0210331.

[23] C. Kristjansen, J. Plefka, G. W. Semenoff and M. Staudacher, Nucl. Phys. B643 (2002) 3, [hepth/0205033; N.R. Constable, D.Z. Freedman, M. Headrick, S. Minwalla, L. Motl, A. Postnikov and W. Skiba, JHEP 0207 (2002) 017 hep-th/0205089].

[24] D. Berenstein, J. Maldacena and H. Nastase, JHEP 0204 (2002) 013 hep-th/0202021. 
[25] J. Ginibre, J. Math. Phys. 6 (1965) 440.

[26] B. Eynard and C. Kristjansen, BMN Correlators by Loop Equations, hep-th/0209244.

[27] R. de Mello Koch, A. Jevicki and J.P. Rodrigues Collective String Field Theory of Matrix Models in the BMN Limit, hep-th/0209155.

[28] M.A. Stephanov, Phys. Rev. Lett. 76 (1996) 4472 [hep-th/9604003].

[29] G. Akemann, Phys. Rev. Lett. 89 (2002) 072002 [hep-th/0204068]; The solution of a chiral random matrix model with complex eigenvalues, Special Issue of J. Phys. A, to appear hep-th/0204246.

[30] G. Akemann, Phys. Rev. D64 (2001) 114021 hep-th/0106053].

[31] G. Akemann, Phys. Lett. B547 (2002) 100 hep-th/0206086.

[32] H. Stahl, V. Totik, General Orthogonal Polynomials, Cambridge University Press, London, 1992.

[33] M.L. Mehta, Random Matrices, Second Edition, Academic Press, London, 1991.

[34] G. Szegö, Orthogonal polynomials, Am. Math. Soc., Colloquium Publications, 23. Providence, 1975.

[35] F. Abild-Pedersen and G. Vernizzi, On the Microscopic Spectra of the Massive Dirac Operator for Chiral Orthogonal and Chiral Symplectic Ensembles, hep-th/0104028.

[36] P. Zinn-Justin, Commun. Math. Phys. 194 (1998) 631 cond-mat/9705044.

[37] G. Akemann and P.H. Damgaard, Nucl. Phys. B576 (2000) 597 [hep-th/9910190].

[38] H.W. Braden, A. Mironov and A. Morozov, Phys. Lett. B514 (2001) 293 hep-th/0105169.

[39] P. Di Francesco, M. Gaudin, C. Itzykson and F. Lesage, Int. J. Mod. Phys. A9 (1994) 4257 hep-th/9401163.

[40] Y.V. Fyodorov, B.A. Khoruzhenko and H.-J. Sommers, Phys. Lett. A226 (1997) 46 condmat/9606173; Phys. Rev. Lett. 79 (1997) 557 cond-mat/9703152. 